\title{
Short-term adverse remodeling progression in asymptomatic aortic stenosis
}

\author{
Anvesha Singh ${ }^{1}$ (D) Daniel C. S. Chan ${ }^{1} \cdot$ Prathap Kanagala $^{1,2} \cdot$ Kai Hogrefe $^{3} \cdot$ Damian J. Kelly $^{4} \cdot$ Jeffery P. Khoo $^{5} \cdot$

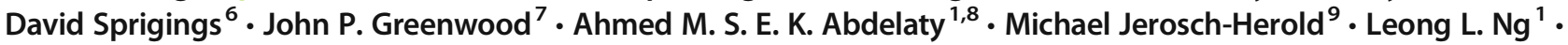 \\ Gerry P. McCann ${ }^{1}$
}

Received: 17 July 2020 / Revised: 7 September 2020 / Accepted: 3 November 2020 / Published online: 19 November 2020

(C) The Author(s) 2020

\begin{abstract}
Objectives Aortic stenosis (AS) is characterised by a long and variable asymptomatic course. Our objective was to use cardiovascular magnetic resonance imaging (MRI) to assess progression of adverse remodeling in asymptomatic AS.

Methods Participants from the PRIMID-AS study, a prospective, multi-centre observational study of asymptomatic patients with moderate to severe AS, who remained asymptomatic at 12 months, were invited to undergo a repeat cardiac MRI.

Results Forty-three participants with moderate-severe AS (mean age $64.4 \pm 14.8$ years, $83.4 \%$ male, aortic valve area index 0.54 $\pm 0.15 \mathrm{~cm}^{2} / \mathrm{m}^{2}$ ) were included. There was small but significant increase in indexed left ventricular (LV) (90.7 \pm 22.0 to $94.5 \pm$ $\left.23.1 \mathrm{ml} / \mathrm{m}^{2}, p=0.007\right)$ and left atrial volumes $\left(52.9 \pm 11.3\right.$ to $\left.58.6 \pm 13.6 \mathrm{ml} / \mathrm{m}^{2}, p<0.001\right)$, with a decrease in systolic (LV ejection fraction $57.9 \pm 4.6$ to $55.6 \pm 4.1 \%, p=0.001$ ) and diastolic (longitudinal diastolic strain rate $1.06 \pm 0.2$ to $0.99 \pm 0.21 / \mathrm{s}$, $p=0.026$ ) function, but no overall change in LV mass or mass/volume. Late gadolinium enhancement increased (2.02 to $4.26 \mathrm{~g}$, $p<0.001$ ) but markers of diffuse interstitial fibrosis did not change significantly (extracellular volume index 12.9 [11.4, 17.0] ml/ $\mathrm{m}^{2}$ to $\left.13.3[11.1,15.1] \mathrm{ml} / \mathrm{m}^{2}, p=0.689\right)$. There was also a significant increase in the levels of NT-proBNP $(43.6[13.45,137.08]$ $\mathrm{pg} / \mathrm{ml}$ to $53.4[19.14,202.20] \mathrm{pg} / \mathrm{ml}, p=0.001)$.

Conclusions There is progression in cardiac remodeling with increasing scar burden even in asymptomatic AS. Given the lack of reversibility of LGE post-AVR and its association with long-term mortality post-AVR, this suggests the potential need for earlier intervention, before the accumulation of LGE, to improve the long-term outcomes in AS.

Key Points

- Current guidelines recommend waiting until symptom onset before valve replacement in severe AS.

- MRI showed clear progression in cardiac remodeling over 12 months in asymptomatic patients with AS, with near doubling in LGE.

- This highlights the need for potentially earlier intervention or better risk stratification in AS.
\end{abstract}

Keywords Aortic valve stenosis · Magnetic resonance imaging · Fibrosis

Anvesha Singh

as707@1e.ac.uk

1 Department of Cardiovascular Sciences, University of Leicester and Cardiovascular Theme, NIHR Leicester Biomedical Research Centre, Glenfield Hospital, Groby road, Leicester LE3 9QP, UK

2 Department of Cardiology, Aintree University Hospital, Liverpool, UK

3 Cardiology Department, Kettering General Hospital Foundation Trust, Rothwell Rd, Kettering NN16 8UZ, UK

4 Cardiology Department, Royal Derby Hospital, Uttoxeter Rd, Derby DE22 3NE, UK
5 Cardiology Department, Glenfield Hospital, Groby Road, Leicester LE3 9QP, UK

6 Northampton General Hospital, Cliftonville, Northampton NN1 $5 \mathrm{BD}, \mathrm{UK}$

7 Multidisciplinary Cardiovascular Research Centre \& The Division of Biomedical Imaging, Leeds Institute of Cardiovascular \& Metabolic Medicine, Leeds University, Leeds LS2 9JT, UK

8 Cardiology Department, Suez Canal University, Ring road, Ismailia 41611, Egypt

9 Brigham and Woman's Hospital and Harvard Medical School, 75 Francis St, Boston, MA 02115, USA 


$\begin{array}{ll}\text { Abbreviations } & \\ \text { AS } & \text { Aortic stenosis } \\ \text { AVA(I) } & \text { Aortic valve area (index) } \\ \text { AVR } & \text { Aortic valve replacement } \\ \text { ECV } & \text { Extracellular volume } \\ \text { iECV } & \text { Absolute extracellular volume index } \\ \text { L/RVEDVI } & \begin{array}{l}\text { Left/right ventricular } \\ \text { end-diastolic volume index }\end{array} \\ \text { L/RVEF } & \text { Left/right ventricle ejection fraction } \\ \text { LA (VI) } & \text { Left atrial (volume index) } \\ \text { LGE } & \text { Late gadolinium enhancement } \\ \text { LVMI } & \text { Left ventricular mass index } \\ \text { MBF } & \text { Myocardial blood flow } \\ \text { MPR } & \text { Myocardial perfusion reserve } \\ \text { MRI } & \text { Magnetic resonance imaging }\end{array}$

\section{Introduction}

Aortic stenosis (AS) is the commonest valve lesion requiring surgery in the developed world, with increasing prevalence with ageing populations [1]. It is characterised by a long and variable asymptomatic course. The development of myocardial fibrosis is key in the transition from compensated hypertrophy to heart failure, with low-grade inflammation also playing a role [2].

Cardiovascular magnetic resonance imaging (MRI) has provided valuable insights into the remodeling patterns in AS [3]. Several MRI-measured markers have been linked to symptoms, exercise capacity and outcome in AS, including myocardial perfusion reserve (MPR) [4, 5]; surrogate markers of diffuse interstitial fibrosis: T1, extracellular volume fraction (ECV) [6], absolute extracellular volume index (iECV) [7]; and extent of focal fibrosis measured by late gadolinium enhancement (LGE) [8]. A large multi-centre study has confirmed LGE to be independently associated with mortality even after aortic valve replacement (AVR) [9]. Others have shown LGE to be irreversible 1-2 years after AVR $[10,11]$, suggesting a need for potentially earlier intervention before LGE is established and the need for potentially reversible markers to identify those for earlier intervention.

Studying the changes in remodeling at an earlier stage of disease may provide important insights into the pathophysiology of disease progression [12]. The aims of this study were to establish the extent of progression in adverse remodeling within 12 months in asymptomatic patients with AS.

\section{Methods}

\section{Subjects}

Asymptomatic patients with moderate to severe AS were recruited as part of the multi-centre, prospective 'PRognostic Importance of MIcrovascular Dysfunction in asymptomatic patients with AS' (PRIMID-AS) study between April 2012 and October $2013[5,13]$. Their asymptomatic status was confirmed by the clinical team whose care they were under, as well as by the patients. Inclusion criteria were ages 18 to 85 years, moderate to severe AS ( $\geq 2$ of aortic valve area $<1.5 \mathrm{~cm}^{2}$, peak pressure gradient $>36 \mathrm{mmHg}$, or mean pressure gradient $>25 \mathrm{mmHg}$ ), asymptomatic, and ability to perform bicycle exercise test. Exclusion criteria were absolute contraindications to MRI/adenosine/contrast, previous cardiac surgery, left ventricular ejection fraction $(\mathrm{LVEF})<40 \%$, persistent atrial flutter/fibrillation, other severe valve disease, previous heart failure, planned AVR or comorbidity limiting life expectancy, or precluding AVR. Those who remained asymptomatic at 12 months on clinical review and direct questioning were invited for a repeat MRI and blood sampling. The UK national research ethics service approved the study (11/EM/0410) and written informed consent was obtained from all participants.

\section{Investigations}

\section{Echocardiography}

A trans-thoracic echocardiogram was performed at baseline by an accredited sonographer according to international guidelines [14]. All image analysis was conducted at the core lab by a single physiologist, using an Xcelera (Phillips) workstation.

\section{MRI}

Patients underwent comprehensive multi-parametric $3 \mathrm{~T}$ cardiac stress MRI including long- and short-axis cine, pre- and post-contrast $\mathrm{T} 1$ mapping, adenosine stress first-pass perfusion imaging, and LGE, at baseline and 12 months, using identical imaging protocol, as previously described [13]. T1 mapping was performed on a single mid-ventricular slice. A full left atrial (LA) and left ventricular (LV) short-axis stack was acquired for volumetric assessment. All image analysis was undertaken at the core lab by a single observer (A.S.), blinded to the patient data. Volumetric, T1, and LGE analyses were performed using cvi42 version 5 (Circle Cardiovascular Imaging). Papillary muscles were excluded from the myocardial mass. LGE was quantified using $>5 \mathrm{SD}$ above the mean signal intensity of normal myocardium [15]. ECV was calculated [16] using haematocrit measured on the same day. iECV $(\mathrm{ECV} \times$ myocardial volume index $)$ and myocyte volume in$\operatorname{dex}([1-\mathrm{ECV}] \times$ myocardial volume index $)$ were calculated 
[17]. To account for a change of flip angle in the $\mathrm{T} 1$ sequence between scans, only ECV-derived measures are shown. Absolute myocardial blood flow (MBF) was calculated using model-independent deconvolution method, using Q-mass version 7.1 (Medis), as previously described [18]. MPR was calculated as the ratio of stress MBF to rest MBF. Diogenes feature tracking software (TomTec Imaging Systems) was used for strain and strain rate analysis [19].

Qualitative LGE assessment was performed by 2 assessors (A.S., G.P.M.) and non-infarct pattern LGE was graded as $0=$ no enhancement, $1=$ mild insertion point enhancement, $2=$ subtle enhancement in 1 region outside insertion point, $3=$ bright scar in 1 region/diffuse enhancement in multiple regions, and $4=$ clear scar in multiple regions (Fig. 1). Typical infarct pattern LGE affecting the subendocardium in a coronary artery territory distribution was also recorded.

\section{Plasma}

Blood samples were collected in EDTA tubes and centrifuged within $4 \mathrm{~h}$ at $2000 \mathrm{~g}$ for $20 \mathrm{~min}$. Plasma was drawn off and stored at $-80{ }^{\circ} \mathrm{C}$. Blinded, single-batch analysis was performed at the end. N-Terminal-pro brain natriuretic peptide (NT-proBNP) was measured using our in-house non-competitive assay that employs the quantitative sandwich enzyme immunoassay technique, and has excellent correlation with the Roche Elecsys assay.

\section{Statistical analysis}

Statistical analysis was performed using SPSS 24.0 software (Statistical Package for the Social Sciences), with $p<0.05$ considered statistically significant. Normally distributed data are expressed as mean \pm standard deviation. Non-parametric data are expressed as median (interquartile range). Continuous variables were compared between baseline and follow-up visit using paired sample $t$ tests or the Wilcoxon signed-rank test. McNemar test was used for categorical variables. Linear regression was used to explore the univariate associations of baseline MRI parameters/echocardiographic Doppler data/ NTproBNP levels with absolute change in the LV remodeling parameters: LV end-diastolic volume index (LVEDVI), LV mass index (LVMI), LV mass/volume, LVEF, left atrial volume index (LAVI) and LGE.

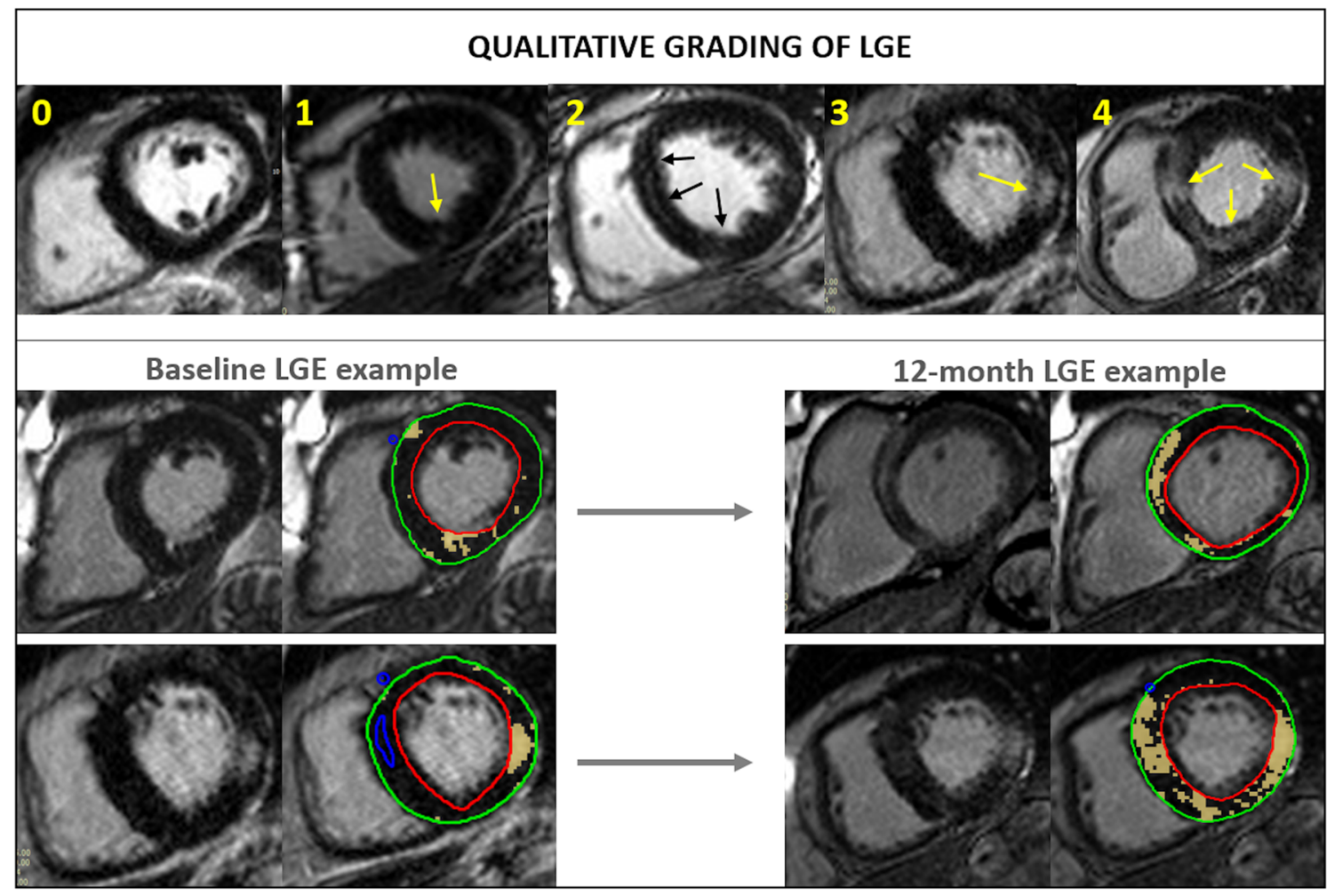

Fig. 1 Qualitative non-infarct pattern LGE grading used is demonstrated in the top panel $(0=$ no enhancement, $1=$ insertion point enhancement, $2=$ subtle enhancement in 1 region outside insertion point, $3=$ bright scar in 1 region/diffuse enhancement in multiple regions, $4=$ clear scar in multiple regions). Typical infarct pattern LGE (subendocardial pattern in a coronary artery territory distribution) was also recorded separately.
Examples of LGE progression over 12 months shown without and with quantification contours (red region of interest (ROI): endocardial contour; green ROI: epicardial contour; blue ROI: area of normal myocardium defined by the user; yellow ROI: automated areas of LGE detected by software using 5 standard deviation method 


\section{Results}

\section{Baseline data}

Forty-three subjects (36 male, age $64.4 \pm 14.8$ years) were included (Table 1). Concomitant hypertension was present in over half the cohort, with diabetes in 4 patients. Most patients had severe AS with an echo-derived aortic valve area index (AVAI) of $0.60 \pm 0.14 \mathrm{~cm}^{2} / \mathrm{m}^{2}$. Bicuspid morphology was present in $40 \%$.

\section{Remodeling at 12 months}

There was no significant change in patients' weight, blood pressure, or heart rate between baseline and follow-up. MRI data is shown in Table 2. Paired T1 maps were available in 25 patients (baseline native T1 $1114.9 \pm 56.7 \mathrm{~ms}$ ) as the T1 mapping sequence was not available from the vendor during parts of the study. Follow-up LGE imaging was acquired in 42 patients. MRI planimetry AVAI remained unchanged at 12 months. There was a significant increase in indexed LV

Table 1 Baseline demographic and echocardiographic data $(n=43)$

\begin{tabular}{lc}
\hline Parameter & Value \\
\hline Age, years & $64.4 \pm 14.8$ \\
Male sex, $n(\%)$ & $36(83.7)$ \\
BMI, $\mathrm{kg} / \mathrm{m}^{2}$ & $28.1 \pm 4.0$ \\
Creatinine & $86.88 \pm 18.0$ \\
Hct & $0.43 \pm 0.03$ \\
Hypertension, $n(\%)$ & $24(55.8)$ \\
Diabetes, $n(\%)$ & $4(9.3)$ \\
Statins, $n(\%)$ & $27(62.8)$ \\
ACE/ARB, $n(\%)$ & $20(46.5)$ \\
Beta-blockers, $n(\%)$ & $12(27.9)$ \\
Ca channel blockers, $n(\%)$ & $11(25.6)$ \\
Moderate AS, $n(\%)$ & $13(30.2)$ \\
Severe AS, $n(\%)$ & $30(69.8)$ \\
Bicuspid valve, $n(\%)$ & $17(39.5)$ \\
AV Vmax, m/s & $3.78 \pm 0.48$ \\
MPG, mmHg & $33.74 \pm 11.30$ \\
AVA, cm ${ }^{2}$ & $1.20 \pm 0.33$ \\
AVAI, cm ${ }^{2} / \mathrm{m}^{2}$ & $0.60 \pm 0.14$ \\
E/A & $0.91 \pm 0.31$ \\
Septal E/e' & $12.8 \pm 3.3$ \\
Lateral E/e' & $9.8 \pm 3.5$ \\
\hline
\end{tabular}

$B M I$ body mass index, $H R$ heart rate, $S B P / D B P$ systolic/diastolic blood pressure, $H c t$ haematocrit, $A C E-I$ angiotensin-converting enzyme inhibitor, $A R B$ angiotensin II receptor blocker, $A V$ Vmax peak aortic jet velocity, $M P G$ mean pressure gradient, $A V A I$ aortic valve area indexed to BSA, $A S$ aortic stenosis, $L G E$ late gadolinium enhancement and LA volumes, with the increase in right ventricular (RV) volumes of borderline statistical significance $(p=0.05)$. These were associated with a small but significant decrease in LV and RV EF, albeit remaining within the normal range. The longitudinal peak diastolic strain rate also decreased significantly. There was a borderline significant increase in LVMI ( $p=0.058)$ but no significant change in LV mass/volume.

\section{Change in measures of fibrosis}

Significant non-infarct pattern LGE (> grade 1 on qualitative analysis) was present in 11 patients at baseline, and 20 at follow-up, with subendocardial infarction in an additional 9 patients at both visits and no new infarctions at follow-up. Excluding those with infarct pattern LGE and with missing follow-up LGE imaging $(n=1)$, the qualitative grade of LGE increased in $39 \%$ (13 out of 33 patients, and in 11 out of 33 to grade 2 or higher) (Figs. 1 and 2), remained unchanged in 52\% $(n=17)$, and decreased by one grade in 3 patients (on blinded analysis). The total amount of LGE(g) doubled over 12 months. There was no significant change in the measures of diffuse interstitial fibrosis: ECV or iECV. There was a decrease in stress and rest MBF, but no change in the MPR.

\section{Plasma biomarker}

There was a significant increase in levels of NTproBNP at 12 months (Table 2). The baseline level of NT-proBNP was significantly correlated with baseline LAVI $(r=0.35, p=0.021)$.

\section{Associations with change in remodeling parameters}

On univariate analysis, baseline LV mass/volume was positively associated with change in LVEDVI, and negatively associated with change in LV mass/volume (Table 3). For change in LVEF, there were significant negative associations with baseline measures of AS severity and LVEF. Baseline LGE, LAVI, native T1, and NT-proBNP associated positively with change in LGE.

\section{Discussion}

In this study, we performed paired MRI and biomarker analysis at baseline and 12 months, in a cohort of patients with significant but asymptomatic AS. We show clear progression in adverse remodeling, with increase in NT-proBNP, despite patients remaining asymptomatic. 
Table 2 MRI and biomarker data at baseline and 12 months

\begin{tabular}{|c|c|c|c|}
\hline Parameter & Baseline & 12 months & $p$ value \\
\hline AVAI, $\mathrm{cm}^{2} / \mathrm{m}^{2}$ & $0.54 \pm 0.15$ & $0.50 \pm 0.11$ & 0.133 \\
\hline LVEDVI, $\mathrm{ml} / \mathrm{m}^{2}$ & $90.7 \pm 22.0$ & $94.5 \pm 23.1$ & 0.007 \\
\hline LVESVI, $\mathrm{ml} / \mathrm{m}^{2}$ & $38.7 \pm 12.3$ & $42.5 \pm 13.6$ & $<0.001$ \\
\hline LVEF, \% & $57.9 \pm 4.6$ & $55.6 \pm 4.1$ & 0.001 \\
\hline LAVI, $\mathrm{ml} / \mathrm{m}^{2}$ & $52.9 \pm 11.3$ & $58.6 \pm 13.6$ & $<0.001$ \\
\hline RVEDVI, $\mathrm{ml} / \mathrm{m}^{2}$ & $89.0 \pm 13.3$ & $92.3 \pm 13.5$ & 0.050 \\
\hline RVEF, \% & $56.8 \pm 6.2$ & $54.5 \pm 4.6$ & 0.006 \\
\hline LVMI, $g / \mathrm{m}^{2}$ & $59.1 \pm 15.1$ & $60.6 \pm 16.0$ & 0.058 \\
\hline LV mass/volume, $\mathrm{g} / \mathrm{ml}$ & $0.66 \pm 0.10$ & $0.64 \pm 0.09$ & 0.231 \\
\hline Septal wall thickness, cm & $1.42 \pm 0.26$ & $1.39 \pm 0.26$ & 0.093 \\
\hline Longitudinal PSS, \% & $-18.5 \pm 2.8$ & $-18.3 \pm 2.4$ & 0.537 \\
\hline Longitudinal PEDSR, 1/s & $1.06 \pm 0.24$ & $0.99 \pm 0.24$ & 0.026 \\
\hline ECV fraction, $\%$ & $24.2 \pm 2.03$ & $24.3 \pm 4.66$ & 0.929 \\
\hline Extracellular volume index, $\mathrm{ml} / \mathrm{m}^{2}$ & $12.9(11.4,17.0)$ & $13.3(11.1,15.1)$ & 0.689 \\
\hline Cellular volume index, $\mathrm{ml} / \mathrm{m}^{2}$ & $42.0(37.4,48.3)$ & $41.5(36.6,48.2)$ & 0.265 \\
\hline Qualitative LGE > grade $1, n(\%)$ & $11(26)$ & $20(48)$ & 0.001 \\
\hline 0 - Normal & 7 & 3 & \\
\hline 1 - Insertion point only & 15 & 10 & \\
\hline 2 - Subtle enhancement in 1 region & 2 & 9 & \\
\hline $\begin{array}{l}3 \text { - Bright scar in } 1 \text { region/diffuse subtle enhance- } \\
\text { ment }\end{array}$ & 7 & 8 & \\
\hline 4 - Scar in multiple regions & 2 & 3 & \\
\hline Infarct pattern & 9 & 9 & \\
\hline LGE, $\mathrm{g}$ & $2.02(1.26,4.57)$ & $4.26(2.17,6.85)$ & $<0.001$ \\
\hline LGE \% LVmass & $2.25(1.03,4.10)$ & $4.20(2.30,6.40)$ & $<0.001$ \\
\hline Rest MBF, ml/min/g & $0.63(0.54,0.78)$ & $0.56(0.44,0.62)$ & $<0.001$ \\
\hline Stress MBF, $\mathrm{ml} / \mathrm{min} / \mathrm{g}$ & $1.34(1.03,1.54)$ & $1.08(0.92,1.34)$ & 0.002 \\
\hline MPR & $2.01 \pm 0.58$ & $2.17 \pm 0.76$ & 0.243 \\
\hline NT-proBNP, pg/mL & $43.6(13.45,137.08)$ & $53.4(19.14,202.20)$ & 0.001 \\
\hline
\end{tabular}

$B M I$ body mass index, $H R$ heart rate, $S B P / D B P$ systolic/diastolic blood pressure, Hct haematocrit, ACE-I angiotensin-converting enzyme inhibitor, $A R B$ angiotensin II receptor blocker, $A V V \max$ peak aortic jet velocity, $M P G$ mean pressure gradient, $A V A I$ aortic valve area indexed to BSA, $A S$ aortic stenosis, $L G E$ late gadolinium enhancement, $L V E D V I$ left ventricular end-diastolic volume index (BSA), LVESVI left ventricular end-systolic volume index, $L V S V I$ left ventricular stroke volume index, $L V E F$ left ventricular ejection fraction, $L V M I$ left ventricular mass index, $L A V I$ left atrial volume index, RVEDVI right ventricular end-diastolic volume index, PSS peak systolic strain, $P E D S R$ peak early diastolic strain rate, $M P R$ myocardial perfusion reserve, $M B F$ myocardial blood flow, $L G E$ late gadolinium enhancement, $E C V$ extracellular volume. Paired T1 mapping available in $n=25$, paired LGE analysis possible in $n=42$, and myocardial blood flow data available in $n=41$

\section{Changes in remodeling}

There was a significant increase in ventricular and LA volumes, with a small decrease in function. There is only one other MRI study assessing cardiac remodeling progression in asymptomatic AS, which showed important differences compared to ours [11]. Their LVMI and wall thickness increased, whilst LVEDVI decreased, with no change in LVEF. However, their cohort of 61 included 26 patients with mild AS, and only 14 with severe AS, compared to ours with majority being severe (30/43). This suggests that at an earlier stage of disease, patients develop more concentric remodeling, whilst ours, at a later stage of disease, switched to more eccentric remodeling, with increase in volumes and a non-significant reduction in wall thickness. Increased concentric remodeling (LV mass/volume) at baseline was associated with greater degree of LV dilatation at 12 months, and negatively associated with change in mass/volume, also suggesting more eccentric remodeling with continued pressure overload caused by AS.

Both systolic and diastolic function decreased, and NTproBNP levels increased, confirming disease progression, despite no significant change in AVA measured on MRI, 


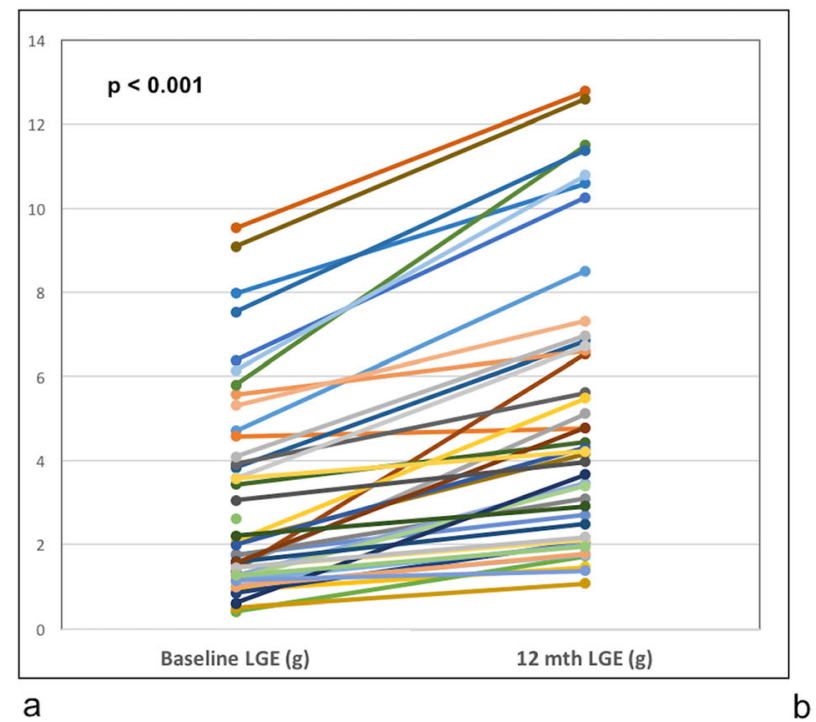

$0=1 \square 2 \square 3 \square 4$

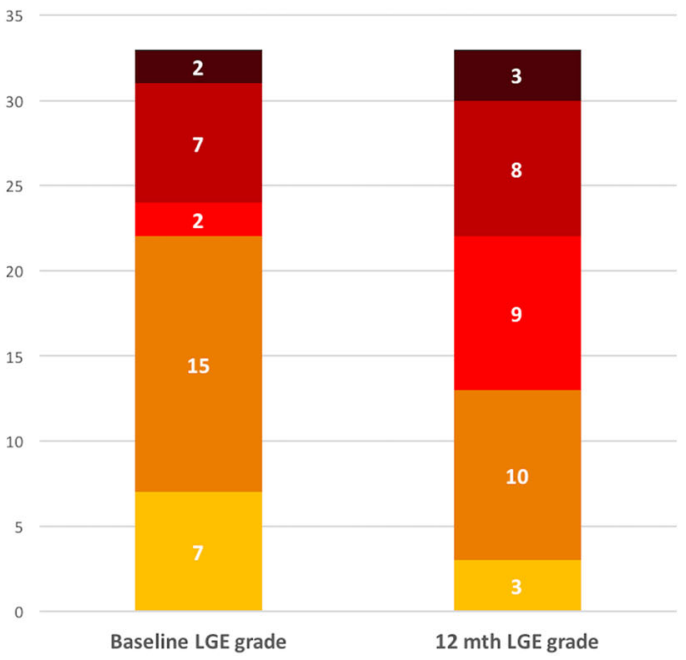

Fig. 2 a Change in LGE from baseline to 12 months. b Qualitative grading of LGE at baseline and 12 months (excluding infarct/no follow-up LGE)

Table 3 Significant univariate associations of baseline parameters with absolute change in LV remodeling parameters on linear regression analysis

\begin{tabular}{llr}
\hline Baseline MRI parameter & Estimate $(95 \% \mathrm{CI})$ & $p$ value \\
\hline $\begin{array}{l}\text { Change in LVEDVI } \\
\text { Baseline LV mass/volume }\end{array}$ & $40.1(15.1,65.2)$ & 0.002 \\
Change in LV mass/volume & & \\
Baseline LV mass/volume & $-0.32(-0.52,-0.12)$ & 0.002 \\
Baseline LAVI & $0.002(0.000,0.004)$ & 0.020 \\
Change in LVEF & & \\
Baseline AV Vmax & $-3.20(-5.76,-0.64)$ & 0.015 \\
Baseline PPG & $-0.10(-0.18,-0.02)$ & 0.018 \\
Baseline MPG & $-0.12(-0.23,-0.01)$ & 0.033 \\
Baseline LVEF & $-0.52(-0.76,-0.29)$ & $<0.001$ \\
Change in LGE (g) & & \\
Baseline LGE (g) & $0.28(0.12,0.45)$ & 0.001 \\
Baseline LAVI & $0.05(0.01,0.08)$ & 0.022 \\
Baseline native T1 & $0.01(0.00,0.02)$ & 0.006 \\
Baseline NT-proBNP & $0.004(0.001,0.007)$ & 0.009 \\
\hline
\end{tabular}

$B M I$ body mass index, $H R$ heart rate, $S B P / D B P$ systolic/diastolic blood pressure, $H c t$ haematocrit, $A C E-I$ angiotensin-converting enzyme inhibitor, $A R B$ angiotensin II receptor blocker, $A V \operatorname{Vmax}$ peak aortic jet velocity, $M P G$ mean pressure gradient, $A V A I$ aortic valve area indexed to BSA, $A S$ aortic stenosis, $L G E$ late gadolinium enhancement, $L V E D V I$ left ventricular end-diastolic volume index (BSA), LVESVI left ventricular endsystolic volume index, $L V S V I$ left ventricular stroke volume index, $L V E F$ left ventricular ejection fraction, $L V M I$ left ventricular mass index, $L A V I$ left atrial volume index, $R V E D V I$ right ventricular end-diastolic volume index, PSS peak systolic strain, PEDSR peak early diastolic strain rate, $M P R$ myocardial perfusion reserve, $M B F$ myocardial blood flow, $L G E$ late gadolinium enhancement, $E C V$ extracellular volume suggesting progressive cardiac decompensation under chronic pressure overload [20]. It is possible that those with more marked progression in AS were intervened on earlier, and therefore, not included in this cohort. Reduced diastolic function is associated with poor prognosis post-AVR [21], and EF $<60 \%$ is associated with disease progression and worse prognosis [22, 23].

\section{Late gadolinium enhancement}

There was marked increase in focal fibrosis. Significant noninfarct pattern LGE (> grade 1) was present in $36 \%$ of all patients at baseline and increased to $57 \%$ at 12 months. A recent meta-analysis showed LGE to be present in $49.6 \%$ of patients with AS [24]. LGE is a poor prognostic marker even after AVR [24, 25] and irreversible once established [10]. However, there was no significant change in ECV, a surrogate of diffuse fibrosis. This may partly be due to T1 mapping only being measured on a single mid-ventricular slice, compared to the full LV being covered for LGE analysis, and the nonischaemic fibrosis often tends to affect the basal slices in early disease. In Everett's study [11], there was also significant increase in LGE of $1.6 \mathrm{~g} /$ year from a similar baseline value of $2.5 \mathrm{~g}$ at baseline, but no change in ECV. Their iECV did increase, due to a corresponding increase in LV mass, which is incorporated in its calculation.

Baseline LGE was positively associated with change in LGE, which is similar to the previous finding of LGE progressing fastest in those with more LGE at baseline [11]. Coronary disease progression is unlikely to be responsible, as there were no new infarcts noted at follow-up. In addition, baseline LAVI and NT-proBNP were also positively correlated with progressive focal fibrosis, again supporting the role of chronic pressure overload caused by AS leading to progressive cardiac decompensation. 


\section{Myocardial blood flow}

Both rest and stress MBF decreased, but there was no change in MPR. MBF is quantified per gram of myocardium, and therefore, this most likely represents an increase in nonmetabolically active fibrotic burden within the myocardium. In this asymptomatic cohort, with exclusion of those who develop symptoms, perfusion falls but remains balanced. Combined with the findings of MPR being a predictor of symptom onset in the original PRIMID study, this suggests that a separation of the rates of decline in rest and stress MBF may be a key step in symptom onset in AS. This hypothesis will of course need to be tested in a separate and larger cohort.

\section{Clinical implications}

Our data highlights the potential need for earlier intervention or development of anti-fibrotic therapy to optimise long-term outcomes in AS. Recent data suggests the non-inferiority of TAVR in lower risk (but still symptomatic severe) AS [26, 27]. The optimal timing of AVR may be even earlier, in the pre-symptomatic stage, prior to establishment of irreversible remodeling. The EVOLVED (NCT03094143), EARLY TAVR (NCT03042104) and EASY-AS (NCT04204915) trials are evaluating such a strategy.

\section{Limitations}

These patients were a subset of those who remained asymptomatic and were not referred for surgery, and comprise a relatively small cohort, although poorly studied previously. T1 mapping data was not available in all patients, and only measured on a single mid-ventricular slice, whilst LGE was measured using a full short-axis stack. Lack of repeat echocardiography for assessment of AS severity at follow-up is another limitation. However, planimetry AVA on CMR has been shown to be a reliable and reproducible technique, with close agreement with AVA on transoesophageal imaging and AS severity on cardiac catheterisation [28-30].

\section{Conclusions}

Asymptomatic patients with moderate to severe AS demonstrate unequivocal progression in adverse cardiac remodeling within 12 months, with a significant increase in focal myocardial fibrosis. Further studies are required to determine whether earlier intervention in asymptomatic AS is associated with improved long-term outcomes.

Acknowledgments We acknowledge the University of Leicester Clinical Trials Unit for providing trial management support.
Funding This study was funded by the National Institute for Health Research (NIHR-PDF 2011-04-51 GPM). The views expressed are those of the authors and not of the NHS, NIHR or the Department of Health. AS was funded by the NIHR Leicester Cardiovascular Biomedical Research Centre. The NIHR Comprehensive Local Research Networks and the Leeds and Leicester NIHR Clinical Research Facilities provided further support. D.C.S.C was funded by the British Heart Foundation (FS/15/10/ 31223 ) and GPM by the NIHR (PDF 2011-04-51, CDF-2014-07-045).

\section{Compliance with ethical standards}

Guarantor The scientific guarantor of this publication is Professor Gerry McCann.

Conflict of interest The authors of this manuscript declare no relationships with any companies whose products or services may be related to the subject matter of the article.

Statistics and biometry No complex statistical methods were necessary for this paper.

Informed consent Written informed consent was obtained from all subjects (patients) in this study.

Ethical approval Institutional Review Board approval was obtained (UK national research ethics service 11/EM/0410).

Study subjects or cohorts overlap This cohort is a sub-set of the main PRIMID-AS study (Eur Heart J. 2017 Apr 21;38(16):1222-1229), which included 174 patients. 43 of these returned for a repeat 12 month MRI scan whilst remaining asymptomatic, which forms the cohort for this manuscript.

\section{Methodology \\ - prospective \\ - observational \\ - multi-centre study}

Open Access This article is licensed under a Creative Commons Attribution 4.0 International License, which permits use, sharing, adaptation, distribution and reproduction in any medium or format, as long as you give appropriate credit to the original author(s) and the source, provide a link to the Creative Commons licence, and indicate if changes were made. The images or other third party material in this article are included in the article's Creative Commons licence, unless indicated otherwise in a credit line to the material. If material is not included in the article's Creative Commons licence and your intended use is not permitted by statutory regulation or exceeds the permitted use, you will need to obtain permission directly from the copyright holder. To view a copy of this licence, visit http://creativecommons.org/licenses/by/4.0/.

\section{References}

1. Nkomo V, Gardin J, Skelton T, Gottdiener J, Scott C, EnriquezSarano M (2006) Burden of valvular heart diseases: a populationbased study. Lancet 368:1005-1011

2. Hein S, Arnon E, Kostin S et al (2003) Progression from compensated hypertrophy to failure in the pressure-overloaded human heart: structural deterioration and compensatory mechanisms. Circulation 107:984-991 
3. Dweck MR, Joshi S, Murigu T et al (2012) Left ventricular remodeling and hypertrophy in patients with aortic stenosis: insights from cardiovascular magnetic resonance. J Cardiovasc Magn Reson 14: 50

4. Steadman CD, Jerosch-Herold M, Grundy B et al (2012) Determinants and functional significance of myocardial perfusion reserve in severe aortic stenosis. J Am Coll Cardiol Img 5:182-189

5. Singh A, Greenwood JP, Berry C et al (2017) Comparison of exercise testing and CMR measured myocardial perfusion reserve for predicting outcome in asymptomatic aortic stenosis: the PRognostic Importance of MIcrovascular Dysfunction in Aortic Stenosis (PRIMID AS) Study. Eur Heart J 38:1222-1229. https://doi.org/ 10.1093/eurheartj/ehx001

6. Flett AS, Sado DM, Quarta G et al (2012) Diffuse myocardial fibrosis in severe aortic stenosis: an equilibrium contrast cardiovascular magnetic resonance study. Eur Heart J Cardiovasc Imaging 13:819-826

7. Chin CWL, Everett RJ, Kwiecinski J et al (2017) Myocardial fibrosis and cardiac decompensation in aortic stenosis. JACC Cardiovasc Imaging 10:1320-1333

8. Dweck MR, Joshi S, Murigu T et al (2011) Midwall fibrosis is an independent predictor of mortality in patients with aortic stenosis. $\mathrm{J}$ Am Coll Cardiol 58:1271-1279

9. Musa TA, Treibel TA, Vassiliou VS et al (2018) Myocardial scar and mortality in severe aortic stenosis: data from the BSCMR Valve Consortium. Circulation. https://doi.org/10.1161/ CIRCULATIONAHA.117.032839

10. Treibel TA, Kozor R, Schofield R et al (2018) Reverse myocardial remodeling following valve replacement in patients with aortic stenosis. J Am Coll Cardiol 71:860-871

11. Everett RJ, Tastet L, Clavel MA et al (2018) Progression of hypertrophy and myocardial fibrosis in aortic stenosis: a multicenter cardiac magnetic resonance study. Circ Cardiovasc Imaging 11: e007451

12. Chin CW, Shah AS, McAllister DA et al (2014) High-sensitivity troponin I concentrations are a marker of an advanced hypertrophic response and adverse outcomes in patients with aortic stenosis. Eur Heart J. https://doi.org/10.1093/eurheartj/ehu189

13. Singh A, Ford I, Greenwood JP et al (2013) Rationale and design of the PRognostic Importance of MIcrovascular Dysfunction in asymptomatic patients with Aortic Stenosis (PRIMID-AS): a multicentre observational study with blinded investigations. BMJ Open 3:e004348

14. Baumgartner H, Hung J, Bermejo J et al (2009) Echocardiographic assessment of valve stenosis: EAE/ASE recommendations for clinical practice. J Am Soc Echocardiogr 22:1-23

15. Flett AS, Hasleton J, Cook C et al (2011) Evaluation of techniques for the quantification of myocardial scar of differing etiology using cardiac magnetic resonance. J Am Coll Cardiol Img 4:150-156

16. Singh A, Horsfield MA, Bekele S, Khan JN, Greiser A, McCann GP (2015) Myocardial T1 and extracellular volume fraction measurement in asymptomatic patients with aortic stenosis: reproducibility and comparison with age-matched controls. Eur Heart J Cardiovasc Imaging 16:763-770

17. McDiarmid AK, Swoboda PP, Erhayiem B et al (2016) Athletic cardiac adaptation in males is a consequence of elevated myocyte mass. Circ Cardiovasc Imaging 9:e003579

18. Jerosch-Herold M, Swingen C, Seethamraju RT (2002) Myocardial blood flow quantification with MRI by model-independent deconvolution. Med Phys 29:886-897

19. Khan JN, Singh A, Nazir SA, Kanagala P, Gershlick AH, McCann GP (2015) Comparison of cardiovascular magnetic resonance feature tracking and tagging for the assessment of left ventricular systolic strain in acute myocardial infarction. Eur J Radiol 84:840-848

20. Steadman CD, Ray S, Ng LL, McCann GP (2010) Natriuretic peptides in common valvular heart disease. J Am Coll Cardiol 55: 2034-2048

21. Dahl JS, Barros-Gomes S, Videbaek L et al (2016) Early diastolic strain rate in relation to systolic and diastolic function and prognosis in aortic stenosis. JACC Cardiovasc Imaging 9:519-528

22. Ito S, Miranda WR, Nkomo VT et al (2018) Reduced left ventricular ejection fraction in patients with aortic stenosis. J Am Coll Cardiol 71:1313-1321

23. Taniguchi T, Morimoto T, Shiomi H et al (2018) Prognostic impact of left ventricular ejection fraction in patients with severe aortic stenosis. JACC Cardiovasc Interv 11:145-157

24. Balciunaite G, Skorniakov V, Rimkus A et al (2020) Prevalence and prognostic value of late gadolinium enhancement on CMR in aortic stenosis: meta-analysis. Eur Radiol 30:640-651

25. Musa TA, Treibel TA, Vassiliou VS et al (2018) Myocardial scar and mortality in severe aortic stenosis. Circulation 138:1935-1947

26. Popma JJ, Deeb GM, Yakubov SJ et al (2019) Transcatheter aorticvalve replacement with a self-expanding valve in low-risk patients. N Engl J Med 380:1706-1715

27. Mack MJ, Leon MB, Thourani VH et al (2019) Transcatheter aortic-valve replacement with a balloon-expandable valve in lowrisk patients. N Engl J Med 380:1695-1705

28. John AS, Dill T, Brandt RR et al (2003) Magnetic resonance to assess the aortic valve area in aortic stenosis: how does it compare to current diagnostic standards? J Am Coll Cardiol 42:519-526

29. Pouleur AC, le Polain de Waroux JB, Pasquet A, Vancraeynest D, Vanoverschelde JL, Gerber BL (2007) Planimetric and continuity equation assessment of aortic valve area: head to head comparison between cardiac magnetic resonance and echocardiography. J Magn Reson Imaging 26:1436-1443

30. Kupfahl C, Honold M, Meinhardt G et al (2004) Evaluation of aortic stenosis by cardiovascular magnetic resonance imaging: comparison with established routine clinical techniques. Heart 90: 893-901

Publisher's note Springer Nature remains neutral with regard to jurisdictional claims in published maps and institutional affiliations. 